\title{
CONVERGENCE TO SPDE OF THE SCHRÖDINGER EQUATION WITH LARGE, RANDOM POTENTIAL*
}

\author{
NINGYAO ZHANG ${ }^{\dagger}$ AND GUILLAUME BAL ${ }^{\ddagger}$
}

\begin{abstract}
We study the asymptotic behavior of solutions to the Schrödinger equation with large-amplitude, highly oscillatory, random potential. In dimension $d<\mathfrak{m}$, where $\mathfrak{m}$ is the order of the leading operator in the Schrödinger equation, we construct the heterogeneous solution by using a Duhamel expansion and prove that it converges in distribution, as the correlation length $\varepsilon$ goes to 0 , to the solution of a stochastic differential equation, whose solution is represented as a sum of iterated Stratonovich integrals, over the space $C\left([0,+\infty), \mathcal{S}^{\prime}\right)$. The uniqueness of the limiting solution in a dense space of $L^{2}\left(\Omega \times \mathbb{R}^{d}\right)$ is shown by verifying the property of conservation of mass for the Schrödinger equation. In dimension $d>\mathfrak{m}$, the solution to the Schrödinger equation is shown to converge in $L^{2}\left(\Omega \times \mathbb{R}^{d}\right)$ to a deterministic Schrödinger solution in [N. Zhang and G. Bal, Stoch. Dyn., 14(1), 1350013, 2014].
\end{abstract}

Key words. Partial differential equation with random coefficients, Duhamel expansion, stochastic partial differential equation, iterated Stratonovich integral.

AMS subject classifications. 35R60, 60H15, 35K15.

\section{Introduction}

We consider the following Schrödinger equation in dimension $d<\mathfrak{m}$ :

$$
\left\{\begin{aligned}
\left(i \frac{\partial}{\partial t}+\left(P(D)-\frac{1}{\varepsilon^{d / 2}} q\left(\frac{x}{\varepsilon}\right)\right)\right) u_{\varepsilon}(t, x) & =0, \quad t>0, x \in \mathbb{R}^{d} \\
u_{\varepsilon}(0, x) & =u_{0}(x), \quad x \in \mathbb{R}^{d}
\end{aligned}\right.
$$

where $P(D)$ is the pseudo-differential operator with symbol $\hat{p}(\xi)=|\xi|^{\mathfrak{m}}$. Taking the Fourier transform of both sides of (1.1), we obtain

$$
\left\{\begin{aligned}
\left(i \frac{\partial}{\partial t}+\xi^{\mathfrak{m}}\right) \hat{u}_{\varepsilon} & =\varepsilon^{-\frac{d}{2}} \int \hat{q}(\zeta) \hat{u}_{\varepsilon}\left(t, \xi-\varepsilon^{-1} \zeta\right) d \zeta \\
\hat{u}_{\varepsilon}(0, \xi) & =\hat{u}_{0}(\xi)
\end{aligned}\right.
$$

We assume that the Fourier transform of the covariance of the potential $\hat{R}(\xi)$ is bounded and continuous at 0 , and the initial condition satisfies $\left(1+|\xi|^{2 \mathfrak{m}}\right)\left|\hat{u}_{0}(\xi)\right| \leq C$ uniformly in $\xi \in \mathbb{R}^{d}$.

The main objective of this paper is to construct a solution to the above equation in $L^{2}\left(\Omega \times \mathbb{R}^{d}\right)$ uniformly in time on bounded intervals and to show that the solution converges in distribution as $\epsilon \rightarrow 0$ to the unique solution of the following stochastic partial differential equation (SPDE):

$$
\left\{\begin{aligned}
i \frac{\partial u}{\partial t}+P(D) u-\sigma u \circ \dot{W} & =0, \quad t>0, x \in \mathbb{R}^{d} \\
u(0, x) & =u_{0}(x), \quad x \in \mathbb{R}^{d}
\end{aligned}\right.
$$

${ }^{*}$ Received: October 26, 2012; accepted (in revised form): June 28, 2013. Communicated by Peter Markowich.

${ }^{\dagger}$ Department of Applied Physics and Applied Mathematics, Columbia University, New York NY, 10027, USA (nz2164@columbia.edu).

${ }^{\ddagger}$ Department of Applied Physics and Applied Mathematics, Columbia University, New York NY, 10027, USA (gb2030@columbia.edu). 
where $\dot{W}$ denotes spatial white noise, o denotes the Stratonovich product, and $\sigma$ is defined as

$$
\sigma^{2}:=(2 \pi)^{d} \hat{R}(0)=(2 \pi)^{d} \int_{\mathbb{R}^{d}} R(x) d x .
$$

To study this solution, we may take the Fourier transform of the equation to get

$$
\begin{aligned}
\left(i \frac{\partial}{\partial t}+\xi^{\mathfrak{m}}\right) \hat{u}(t, \xi) & =\sigma(2 \pi)^{-d} \int e^{-i \xi x} u(s, x) \circ d W(x) \\
& =\sigma(2 \pi)^{-d} \int e^{-i \xi x} \int e^{i \xi_{1} x} \hat{u}\left(s, \xi_{1}\right) d \xi_{1} \circ d W(x),
\end{aligned}
$$

with initial condition $\hat{u}(0, \xi)=\hat{u}_{0}(\xi)$. To look for a mild solution, we recast (1.5) as

$$
\hat{u}=i \sigma(2 \pi)^{-d} \iint_{0}^{t} e^{i \xi^{\mathfrak{m}}(t-s)} e^{-i \xi x} \int e^{i \xi_{1} x} \hat{u}\left(s, \xi_{1}\right) d \xi_{1} d s \circ d W(x)+e^{i t \xi^{\mathfrak{m}}} \hat{u}_{0}(\xi) .
$$

Define formally the stochastic integral

$$
\mathcal{H} \hat{u}(t, \xi)=(-i \sigma)(2 \pi)^{-d} \int_{0}^{t} e^{i(t-s) \xi^{\mathfrak{m}}} \int e^{-i \xi x} \int e^{i \xi_{1} x} \hat{u}\left(s, \xi_{1}\right) d \xi_{1} d s \circ d W(x) .
$$

We may rewrite (1.6) as

$$
\hat{u}(t, \xi)=e^{i t \xi^{\mathfrak{m}}} \hat{u}_{0}(\xi)+\mathcal{H} \hat{u}(t, \xi) .
$$

The mild solution to (1.3) is thus defined as $u(t, x)=\mathcal{F}^{-1}\{\hat{u}(t, \xi)\}$, where $\mathcal{F}^{-1}$ denotes the inverse Fourier transform. Suppose that $d<\mathfrak{m}$. The following result holds for the solution of (1.5).

TheOREm 1.1. Suppose $d<\mathfrak{m}$. The series

$$
\hat{u}(t, \xi):=\sum_{n \geq 0} \hat{u}^{(n)}(t, \xi)
$$

converges in the $L^{2}\left(\Omega \times \mathbb{R}^{d}\right)$ sense for each $t \geq 0$ and $\xi \in \mathbb{R}^{d}$, and is the unique solution to (1.5) in the space $M$, dense in $L^{2}\left(\Omega \times \mathbb{R}^{d}\right)$ and defined in Section 5, where

$$
\begin{aligned}
\hat{u}^{(n)}= & (-i \sigma)^{n}(2 \pi)^{-n d} e^{t} \int_{0}^{\infty} \cdots \int_{0}^{\infty} \int e^{i \beta t} d \beta \int \prod_{k=1}^{n} d \xi_{k} \\
& \left\{\prod_{k=0}^{n}\left[1-i\left(\left|\xi-\sum_{j=1}^{k} \xi_{j}\right|^{\mathfrak{m}}-\beta\right)\right]\right\} \prod_{j=1}^{n} e^{-i \xi_{j} x_{j}} \hat{u}_{0}\left(\xi-\sum_{j=1}^{n} \xi_{j}\right) \circ \prod_{j=1}^{n} d W\left(\xi_{j}\right) .
\end{aligned}
$$

The following theorem shows the weak convergence of $u_{\varepsilon}(t, x)$ to $u(t, x)$ for any $t>0$ and $x \in \mathbb{R}^{d}$.

TheOREM 1.2. Suppose that $d<\mathfrak{m}$. For any integers $r \geq 1, m_{1}, \cdots, m_{r} \geq 0$ and $\xi^{(1)}, \cdots, \xi^{(r)} \in \mathbb{R}^{d}, t_{1}, \cdots, t_{r} \geq 0$ we have the convergence of moments

$$
\lim _{\varepsilon \rightarrow 0_{+}} \mathbb{E}\left\{\left[\hat{u}_{\varepsilon}\left(t_{1}, \xi^{(1)}\right)\right]^{m_{1}} \cdots\left[\hat{u}_{\varepsilon}\left(t_{r}, \xi^{(r)}\right)\right]^{m_{r}}\right\}=\mathbb{E}\left\{\left[\hat{u}\left(t_{1}, \xi^{(1)}\right)\right]^{m_{1}} \cdots\left[\hat{u}\left(t_{r}, \xi^{(r)}\right)\right]^{m_{r}}\right\} .
$$


The finite dimensional distribution of $\hat{u}(t, \xi)$ is uniquely determined by its moments of all orders. Moreover, the family of processes $\left\{\hat{u}_{\varepsilon}(t, \cdot), t \geq 0\right\}$ is tight, as $\varepsilon \rightarrow 0_{+}$, over $C\left([0,+\infty) ; \mathcal{S}^{\prime}\left(\mathbb{R}^{d}\right)\right)$. The process $\left\{\hat{u}_{\varepsilon}(t, \cdot), t \geq 0\right\}$ converges in law over $C\left([0,+\infty) ; \mathcal{S}^{\prime}\left(\mathbb{R}^{d}\right)\right)$, as $\varepsilon \rightarrow 0_{+}$, to $\{\hat{u}(t, \cdot), t \geq 0\}$. Also, we have that in the spatial domain, the process $\left\{u_{\varepsilon}(t, \cdot), t \geq 0\right\}$ converges in law to $\{u(t, \cdot), t \geq 0\}$.

The rest of the paper is structured as follows. Section 2 gives the formal Duhamel solutions for both the multi-scale Schrödinger equation and the limiting SPDE in the Fourier domain. Section 3 demonstrates the first order moment convergence of Duhamel solutions $\hat{u}_{\varepsilon}$. Section 4 proves that $\hat{u}$, as the Duhamel expansion of the limiting equation, is well defined in the space of in $L^{2}\left(\Omega \times \mathbb{R}^{d}\right)$. Section 5 generalizes the first order moment convergence proved in Section 3 to arbitrary orders. Section 6 follows the approach as in [5] to show that the weak convergence of $\left\{\hat{u}_{\varepsilon}(t, \xi)\right\}$ in $C\left([0,+\infty), \mathcal{S}^{\prime}\right)$ to $\hat{u}(t, \xi)$ follows from tightness and convergence in finite dimensional distribution.

The asymptotic theory of solution to parabolic equation with large potential in dimension $d<\mathfrak{m}$ is presented in [1,6]. In [5], analysis is provided of the heat equation with long range correlated potential in $d=3$. Equation (1.1) may be seen as a model for quantum dynamics with the wavelength of initial condition much larger than the scale of oscillations of the random potential. The treatment of Schrödinger equation (1.1) with the right scaling of potential $\left(O\left(\varepsilon^{-\frac{\mathfrak{m}}{2}}\right)\right)$, in dimension $d>\mathfrak{m}$, is presented in [7]. It is then shown that $u_{\varepsilon}$ converges in $L^{2}\left(\Omega \times \mathbb{R}^{d}\right)$ to the solution of a homogenized equation. For the case $d=\mathfrak{m}$ a logarithmic correction to the scaling of potential appears, which causes the solution to (1.1) to be a deterministic solution to a homogenized equation. Although this critical dimension case which separates homogenization from a stochastic limit is not discussed in [7], it is analyzed in [2] for parabolic equations, to which we refer the readers for more details.

\section{Duhamel expansion}

Iterating Duhamel's formula we obtain

$$
\begin{aligned}
\hat{u}_{\varepsilon}(t, \xi) & =e^{i \xi^{\mathfrak{m}} t} \hat{u}_{0}(\xi)-i \varepsilon^{-\frac{d}{2}} \int_{0}^{t} \int e^{i \xi^{\mathfrak{m}}(t-s)} \hat{q}(\zeta) \hat{u}_{\varepsilon}\left(s, \xi-\varepsilon^{-1} \zeta\right) d s d \zeta \\
& =\sum_{n=0}^{+\infty} \hat{u}_{\varepsilon}^{(n)}(t, \xi)
\end{aligned}
$$

where

$$
\begin{aligned}
\hat{u}_{\varepsilon}^{(n)}(t, \xi)=(-i)^{n} \varepsilon^{-\frac{n d}{2}} \int \cdots \int_{\Delta_{n}(t)} d s_{1} \cdots d s_{n} \int & \cdots \int_{k=1}^{n+1} e^{i\left(s_{k-1}-s_{k}\right)\left|\xi-\varepsilon^{-1} \sum_{j=1}^{k-1} \xi_{j}\right|^{\mathrm{m}}} \\
& \prod_{k=1}^{n} \hat{q}\left(\xi_{k}\right) d \xi_{k} \hat{u}_{0}\left(\xi-\varepsilon^{-1} \sum_{j=1}^{n} \xi_{j}\right)
\end{aligned}
$$

Here, we introduce the notation $\sum_{j=1}^{0} \xi_{j}:=0$ and $\Delta_{n}(t):=\left[t \geq s_{1} \geq \cdots \geq s_{n} \geq 0\right]$. Let $\tilde{\Delta}_{n}(t):=\left[\sum_{j=1}^{n} \tau_{j} \leq t, \tau_{j} \geq 0\right]$. Changing variables $s_{j}:=\sum_{i=j}^{n} \tau_{i}$ and denoting $\tau_{0}:=t-$ $\sum_{i=1}^{n} \tau_{i}$ we can rewrite $(2.2)$ in the form

$$
\hat{u}_{\varepsilon}^{(n)}(t, \xi)=(-i)^{n} \varepsilon^{-\frac{n d}{2}} \int \cdots \int_{\tilde{\Delta}_{n(t)}} d \tau_{1} \cdots d \tau_{n} \int \cdots \int \prod_{k=1}^{n} \hat{q}\left(\xi_{k}\right) d \xi_{k}
$$




$$
\begin{aligned}
& \times \prod_{k=1}^{n+1} e^{i \tau_{k-1}\left|\xi-\varepsilon^{-1} \sum_{j=1}^{k-1} \xi_{j}\right|^{\mathrm{m}}} \hat{u}_{0}\left(\xi-\varepsilon^{-1} \sum_{j=1}^{n} \xi_{j}\right) \\
=(-i)^{n} & \varepsilon^{-\frac{n d}{2}} \int_{0}^{+\infty} \cdots \int_{0}^{+\infty} \int \cdots \int d \tau_{0} \cdots d \tau_{n} \delta\left(t-\sum_{j=0}^{n} \tau_{j}\right) \prod_{k=1}^{n} \hat{q}\left(\xi_{k}\right) d \xi_{k} \\
& \times \prod_{k=1}^{n+1} e^{i \tau_{k-1}\left|\xi-\varepsilon^{-1} \sum_{j=1}^{k-1} \xi_{j}\right|^{\mathrm{m}}}
\end{aligned}
$$

Using $\delta(t)=\int e^{i \beta t} d \beta$, we obtain, for any $\eta>0$,

$$
\begin{aligned}
\hat{u}_{\varepsilon}^{(n)}(t, \xi)=( & -i)^{n} \varepsilon^{-\frac{n d}{2}} e^{\eta t} \int_{0}^{+\infty} \cdots \int_{0}^{+\infty} \int \cdots \int d \tau_{0} \cdots d \tau_{n} \prod_{k=1}^{n} \hat{q}\left(\xi_{k}\right) d \xi_{k} \\
& \times e^{i \beta\left(t-\sum_{j=0}^{n} \tau_{j}\right)} \prod_{k=1}^{n+1} e^{i \tau_{k-1}\left|\xi-\varepsilon^{-1} \sum_{j=1}^{k-1} \xi_{j}\right|^{\mathfrak{m}}} \hat{u}_{0}\left(\xi-\varepsilon^{-1} \sum_{j=1}^{n} \xi_{j}\right) .
\end{aligned}
$$

Integrating out all $\tau_{j}$ and choosing $\eta=1$ we get

$$
\begin{aligned}
\hat{u}_{\varepsilon}^{(n)}(t, \xi)=( & -i)^{n} \varepsilon^{-\frac{n d}{2}} e^{\eta t} \int_{\mathbb{R}} e^{i \beta t} d \beta \int \ldots \int \prod_{k=1}^{n} \hat{q}\left(\xi_{k}\right) d \xi_{k} \\
& \times\left\{\prod_{k=0}^{n}\left[1-i\left(\left|\xi-\varepsilon^{-1} \sum_{j=1}^{k} \xi_{j}\right|^{\mathfrak{m}}-\beta\right)\right]\right\}^{-1} \hat{u}_{0}\left(\xi-\varepsilon^{-1} \sum_{j=1}^{n} \xi_{j}\right) .
\end{aligned}
$$

We now come to the analysis of the limiting equation. By Duhamel's principle, the solution to (1.5) formally satisfies the equation

$$
\begin{aligned}
\hat{u}(t, \xi) & =e^{i \xi^{\mathfrak{m}} t} \hat{u}_{0}(\xi)+(-i \sigma)(2 \pi)^{-d} \int_{0}^{t} e^{i(t-s) \xi^{\mathfrak{m}}} \int e^{-i \xi x} u(s, x) d s \circ d W(x) \\
& =e^{i \xi^{\mathfrak{m}} t} \hat{u}_{0}(\xi)+(-i \sigma)(2 \pi)^{-d} \int_{0}^{t} e^{i(t-s) \xi^{\mathfrak{m}}} \int e^{-i \xi x} \int e^{i \xi_{1} x} \hat{u}\left(s, \xi_{1}\right) d \xi_{1} d s \circ d W(x) .
\end{aligned}
$$

Integrating (2.6) iteratively, we obtain formally the Duhamel expansion for (1.5):

$$
\hat{u}(t, \xi)=\sum_{n=0}^{\infty} \hat{u}^{(n)},
$$

where

$$
\hat{u}^{(0)}=e^{i t \xi^{\mathrm{m}}} \hat{u}_{0}(\xi) \quad \text { and } \quad \hat{u}^{(n)}=\mathcal{H}^{n} \hat{u}^{(0)},
$$

for $n=0,1, \cdots$, or more explicitly,

$$
\begin{aligned}
\hat{u}^{(n)}=(-i \sigma)^{n}(2 \pi)^{-n d} & \cdots \int_{\Delta_{n}(t)} \iint \prod_{k=1}^{n+1} e^{i\left(s_{k-1}-s_{k}\right) \xi_{k-1}^{\mathrm{m}}} \\
& \prod_{j=1}^{n} e^{-i\left(\xi_{j}-\xi_{j-1}\right) x_{j}} \hat{u}_{0}\left(\xi_{n}\right) \prod_{k=1}^{n} d \xi_{k} \prod_{k=1}^{n} d s_{k} \circ \prod_{j=1}^{n} d W\left(x_{j}\right) .
\end{aligned}
$$


Here, we define $s_{0}=t, s_{n+1}=0$, and $\xi_{0}:=\xi$. At this point neither the iterative Stratonovich integral of $\hat{u}^{(n)}$ for $n=1,2, \cdots$ in (2.9) nor the sum (2.7) are well defined. We give a justification for these expressions in Section 4 .

Using the change of variables $\xi_{k} \rightarrow \xi_{k-1}-\xi_{k}$, and applying the same type of transform as in (2.5), we obtain

$$
\begin{aligned}
\hat{u}^{(n)}=(-i \sigma)^{n}(2 \pi)^{-n d} e^{t} \int_{\mathbb{R}^{d}} \int \cdots \int e^{i \beta t} d \beta \int \prod_{k=1}^{n} d \xi_{k} \\
\quad\left\{\prod_{k=0}^{n}\left[1-i\left(\left|\xi-\sum_{j=1}^{k} \xi_{j}\right|^{\mathfrak{m}}-\beta\right)\right]\right\} \\
\prod_{j=1}^{-1} e^{-i \xi_{j} x_{j}} \hat{u}_{0}\left(\xi-\sum_{j=1}^{n} \xi_{j}\right) \circ \prod_{j=1}^{n} d W\left(x_{j}\right),
\end{aligned}
$$

which is the same as (1.10).

\section{Convergence of the first moments}

We shall prove the convergence of moments as stated in (1.11). For simplicity, we shall first consider the case when $n=m=1$ and show that the limit

$$
\lim _{\varepsilon \rightarrow 0_{+}} \mathbb{E} \hat{u}_{\varepsilon}(t, \xi)
$$

exists. The proof of convergence for general moments is given in Section 6 .

Taking expectation of both sides of (2.1), we obtain

$$
\mathbb{E} \hat{u}_{\varepsilon}(t, \xi)=\sum_{n \geq 0} \mathbb{E} \hat{u}_{\varepsilon}^{(2 n)}(t, \xi) .
$$

This is because expectation of the product of an odd number of Gaussian random variables is 0 . The expectation of the product of an even number of Gaussian random variables is given as a sum of products of the expectation of pairs of variables, where the summation runs over all possible pairs. The contribution of products of potentials can thus be represented by

$$
\mathbb{E}\left\{\prod_{k=1}^{2 n} \hat{q}\left(\xi_{k}\right)\right\}=\sum_{\pi} \prod_{(e f) \in \pi} \hat{R}\left(\xi_{e}\right) \delta\left(\xi_{e}+\xi_{f}\right),
$$

where $(e f)$ denotes pair of indices, $\pi$ denotes a pairing of the $2 n$ indices, and the summation is over all possible pairings.

Adding up all the delta functions gives $\sum_{k=1}^{2 n} \xi_{k}=0$. We can therefore write

$$
\begin{aligned}
& \mathbb{E} \hat{u}_{\varepsilon}^{(2 n)}(t, \xi) \\
=(-1)^{n} \varepsilon^{n d} e^{t} \hat{u}_{0}(\xi) \int_{\mathbb{R}} e^{i \beta t} d \beta \int \cdots \int & \\
\times & \left\{\prod_{k=0}^{2 n}\left[1-i\left(\left|\xi-\varepsilon^{-1} \sum_{j=1}^{k} \xi_{j}\right|^{\mathfrak{m}}-\beta\right)\right]\right\}^{-1} \mathbb{E}\left\{\prod_{k=1}^{2 n} \hat{q}\left(\xi_{k}\right) d \xi_{k}\right\} \\
=(-1)^{n} \varepsilon^{n d} e^{t} \hat{u}_{0}(\xi) \sum_{\pi} \int_{\mathbb{R}} e^{i \beta t} d \beta \int \ldots \int & \\
\times & \left\{\prod_{k=0}^{2 n}\left[1-i\left(\left|\xi-\varepsilon^{-1} \sum_{j=1}^{k} \xi_{j}\right|^{\mathfrak{m}}-\beta\right)\right]\right\}^{-1} \prod_{(e f) \in \pi} \hat{R}\left(\xi_{e}\right) \delta\left(\xi_{e}+\xi_{f}\right) d \xi_{e} d \xi_{f} .
\end{aligned}
$$


The above summation extends over all possible pairings $\pi$ made over vertices $\{1, \cdots, 2 n\}$. By changing variables $\xi_{k}:=\varepsilon^{-1} \xi_{k}$, we obtain

$$
\begin{aligned}
\mathbb{E} \hat{u}_{\varepsilon}^{(2 n)}(t, \xi)=(-1)^{n} e^{t} \hat{u}_{0}(\xi) \sum_{\pi} \int_{\mathbb{R}} e^{i \beta t} d \beta \int \cdots \int \\
\quad \times\left\{\prod_{k=0}^{2 n}\left[1-i\left(\left|\xi-\sum_{j=1}^{k} \xi_{j}\right|^{\mathfrak{m}}-\beta\right)\right]\right\}^{-1} \prod_{(e f) \in \pi} \hat{R}\left(\xi_{e}\right) \delta\left(\xi_{e}+\xi_{f}\right) d \xi_{e} d \xi_{f} .
\end{aligned}
$$

We will show that the exchange of taking limit and expectation,

$$
\lim _{\varepsilon \rightarrow 0_{+}} \mathbb{E} \hat{u}_{\varepsilon}(t, \xi)=\sum_{n=0}^{+\infty} \lim _{\varepsilon \rightarrow 0_{+}} \mathbb{E} \hat{u}_{\varepsilon}^{(2 n)}(t, \xi)
$$

is legitimate based on the fact that the sequence $\left\{\sup \left|\mathbb{E} \hat{u}_{\varepsilon}^{(2 n)}(t, \xi)\right|\right\}_{n=1}^{\infty}$ is summable. Let $\mathcal{L}(\pi)$ be the set of all left vertices of a given pairing $\pi$. Define

$$
A_{k}:=\left|\xi-\sum_{j=1}^{k} \xi_{j}\right|^{\mathfrak{m}}
$$

and

$$
\hat{e}_{A}^{(\rho)}=[1-i(A-\beta)]^{-1} .
$$

Using the contour integration method, we are able to show that

$$
e_{A}^{(\rho)}= \begin{cases}e^{i A t} t^{\rho-1} e^{-t}, & t>0 \\ 0, & t<0 .\end{cases}
$$

The following estimate is then derived.

Lemma 3.1. Suppose that $\rho>0$. There exists a constant $C>0$ such that for an arbitrary $n \geq 1$ we have

$$
\left|\int e^{i \beta t}\left\{\prod_{k=1}^{n}\left[1-i\left(A_{k}-\beta\right)\right]\right\}^{-\rho} d \beta\right| \leq \frac{C^{n} t^{n \rho-1} e^{-t}}{[(n-1) !]^{\rho}} .
$$

Readers are referred to [5] for the proof of this lemma.

With such notation, we may rewrite (3.4) as

$$
\begin{gathered}
\mathbb{E} \hat{u}_{\varepsilon}^{(2 n)}(t, \xi)=(-1)^{n} e^{t} \hat{u}_{0}(\xi) \sum_{\pi} \int \cdots \int_{k \notin \mathcal{L}(\pi), k \neq 2 n} * \prod_{A_{k}} * F(t, \boldsymbol{\xi} ; \pi) \\
\times \prod_{(e f) \in \pi} \hat{R}\left(\varepsilon \xi_{e}\right) \delta\left(\xi_{e}+\xi_{f}\right) d \xi_{e} d \xi_{f}
\end{gathered}
$$

where

$$
F(t, \boldsymbol{\xi} ; \pi):=\int e^{i \beta t}\left(1-i\left(\xi^{\mathfrak{m}}-\beta\right)\right)^{-2} \prod_{k \in \mathcal{L}(\pi)}\left[1-i\left(\left|\xi-\sum_{j=1}^{k} \xi_{j}\right|^{\mathfrak{m}}-\beta\right)\right]^{-1} d \beta
$$


and $\prod_{k \notin \mathcal{L}(\pi), k \neq 2 n} * \hat{e}_{A_{k}}$ denotes the convolution of all $e_{A_{k}}$ 's, where $k$ is the right vertices in the giving pairing $\pi$, except $k=2 n$.

The following inequality plays an important role in estimating $\mathbb{E} \hat{u}_{\varepsilon}^{(2 n)}(t, \xi)$.

Lemma 3.2. Suppose $\rho \in\left(\frac{d}{\mathfrak{m}}, 1\right)$. Then

$$
\sup _{\beta \in \mathbb{R}, \omega \in \mathbb{R}^{d}} \int_{\mathbb{R}^{d}}|-\beta+| \xi-\left.\omega\right|^{\mathfrak{m}}+\left.i\right|^{-\rho} d \xi<+\infty
$$

Proof. We may first shift $\xi$ to get rid of $\omega$ and perform the spherical change of coordinates

$$
\int_{\mathbb{R}^{d}}|-\beta+| \xi-\left.\omega\right|^{\mathfrak{m}}+\left.i\right|^{-\rho} \leq \Omega_{d} \int_{0}^{+\infty}\left|-\beta+\xi^{\mathfrak{m}}+i\right|^{-\rho} \xi^{d-1} d|\xi|,
$$

where $\Omega_{d}$ is the area of the unit sphere in $\mathbb{R}^{d}$. Let $Q=\xi^{\mathfrak{m}}$. The integral on the right hand side of (3.13) may be rewritten as

$$
\int_{0}^{+\infty}|-\beta+Q+i|^{-\rho} Q^{d / \mathfrak{m}-1} d Q
$$

Without loss of generality, we assume $\beta \geq 0$ and the integral above can then be written as the sum of three integrals $I, I I, I I I$ according to whether $\xi$ belongs to $(0, \beta / 2)$, $(\beta / 2,2 \beta)$, or $(2 \beta,+\infty)$. We have

$$
I \leq\left[1+\left(\frac{\beta}{2}\right)^{2}\right]^{-\frac{\rho}{2}} \int_{0}^{\beta / 2} Q^{\frac{d}{\mathfrak{m}}-1} d Q \leq \frac{\mathfrak{m}}{d}\left[1+\left(\frac{\beta}{2}\right)^{2}\right]^{-\frac{\rho}{2}}\left(\frac{\beta}{2}\right)^{\frac{d}{\mathfrak{m}}} \leq \frac{\mathfrak{m}}{d}
$$

The second integral can also be estimated as

$$
I I \leq(2 \beta)^{\frac{d}{m}-1} \int_{\frac{\beta}{2}}^{2 \beta}|Q-\beta+i|^{-\rho} d Q .
$$

If $\beta \leq 1$ we have

$$
I I \leq 3 \times 2^{\frac{d}{\mathrm{~m}}-2} .
$$

If $\beta>1$, we estimate

$$
I I \leq(2 \beta)^{\frac{d}{m}-1} \int_{\frac{\beta}{2}}^{2 \beta}|Q-\beta|^{-\rho} d Q \leq\left[1+\left(\frac{1}{2}\right)^{1-\rho}\right] 2^{\frac{d}{\mathfrak{m}}-1} .
$$

The third integral is estimated as

$$
I I I \leq \frac{\mathfrak{m}}{d}\left(\beta^{2}+1\right)^{-\frac{\rho}{2}} \beta^{\frac{d}{\mathfrak{m}}} \leq \frac{\mathfrak{m}}{d} .
$$

This concludes the proof of the lemma.

Let

$$
F_{1}(t, \boldsymbol{\xi} ; \pi):=\int_{\mathbb{R}} \frac{e^{i \beta t} d \beta}{\left[1-i\left(\xi^{\mathfrak{m}}-\beta\right)\right]^{2}}\left\{\prod_{k \in \mathcal{L}(\pi)}\left[1-i\left(\left|\xi-\sum_{j=1}^{k} \xi_{j}\right|^{\mathfrak{m}}-\beta\right)\right]^{-\rho}\right\}
$$


and

$$
F_{2}(t, \boldsymbol{\xi} ; \pi):=\int_{\mathbb{R}} e^{i \beta t} d \beta\left\{\prod_{k \in \mathcal{L}(\pi)}\left[1-i\left(\left|\xi-\sum_{j=1}^{k} \xi_{j}\right|^{\mathfrak{m}}-\beta\right)\right]^{-(1-\rho)}\right\} .
$$

Since

$$
F_{1}(t, \boldsymbol{\xi} ; \pi)=e_{B_{1}(\boldsymbol{\xi})}^{(\rho)} * \cdots * e_{B_{n+2}(\boldsymbol{\xi})}^{(\rho)}(t)
$$

for some $B_{j}(\boldsymbol{\xi})$, we get $F_{1}(t, \boldsymbol{\xi} ; \pi)=0$ for $t<0$. Likewise, $F_{2}(t, \boldsymbol{\xi} ; \pi)=0$ for $t<0$. We can therefore write

$$
F(t, \boldsymbol{\xi} ; \pi)=\int_{0}^{t} F_{1}(t-s, \boldsymbol{\xi} ; \pi) F_{2}(s, \boldsymbol{\xi} ; \pi) d s \geq 0 .
$$

Observe that by Lemma 3.1,

$$
F_{2}(t, \boldsymbol{\xi} ; \pi) \leq \frac{C^{n} t^{n(1-\rho)-1}}{[(n-1) !]^{1-\rho}}
$$

and

$$
\prod_{k \notin \mathcal{L}(\pi), k \neq 2 n} * e_{A_{k}}(t) \leq \frac{C^{n} t^{n-1}}{(n-1) !}
$$

for $t>0$. On the other hand, we have obviously

$$
\left|F_{1}(t, \boldsymbol{\xi} ; \pi)\right| \leq C \int_{\mathbb{R}} \frac{d \beta}{1+\left|\xi^{\mathfrak{m}}-\beta\right|^{2}}\left\{\prod_{k \in \mathcal{L}(\pi)}\left|1-i\left(\left|\xi-\sum_{j=1}^{k} \xi_{j}\right|^{\mathfrak{m}}-\beta\right)\right|^{-\rho}\right\}
$$

for some constant $C>0$. As a result, we obtain

$$
\begin{aligned}
& \left|\mathbb{E} \hat{u}_{\varepsilon}^{(2 n)}(t, \xi)\right| \\
\leq & C e^{t}\left|\hat{u}_{0}(\xi)\right| \sum_{\pi} \int_{0}^{t} \frac{(t-s)^{n-1} d s}{(n-1) !} \times \int \ldots \int F(s, \xi ; \pi) \prod_{(k l) \in \pi} \hat{R}\left(\varepsilon \xi_{e}\right) \delta\left(\xi_{e}+\xi_{f}\right) d \xi_{e} d \xi_{f} \\
\leq & \frac{C^{n} e^{t}\left|\hat{u}_{0}(\xi)\right|}{[(n-1) !]^{2-\rho}} \int_{0}^{t}(t-s)^{n} s^{n(1-\rho)-1} d s \sum_{\pi} \int_{\mathbb{R}} \frac{d \beta}{1+\left|\xi^{\mathfrak{m}}-\beta\right|^{2}} \int \ldots \int \\
\quad & \quad \prod_{(e f) \in \pi} \hat{R}\left(\varepsilon \xi_{e}\right) \delta\left(\xi_{e}+\xi_{f}\right) d \xi_{e} d \xi_{f}\left\{\prod_{k \in \mathcal{L}(\pi)}\left|1-i\left(\left|\xi-\sum_{j=1}^{k} \xi_{j}\right|^{\mathfrak{m}}-\beta\right)\right|^{-\rho}\right\}
\end{aligned}
$$

Here, we just need half of the order of decay of the initial condition $\hat{u}_{0}(\xi)$ that we assumed:

$$
\frac{\left|\hat{u}_{0}(\xi)\right|}{\left(1+\left|\xi^{\mathfrak{m}}-\beta\right|^{2}\right)^{1 / 2}} \leq \frac{C}{\left(1+\xi^{2 \mathfrak{m}}\right)^{1 / 2}\left(1+\left|\xi^{\mathfrak{m}}-\beta\right|^{2}\right)^{1 / 2}} \leq \frac{1}{\left(1+\beta^{2}\right)^{1 / 2}} .
$$

The right hand side of the above inequality is then used for estimating the integration in $\beta$ by Lemma 5.2 in [7]:

$$
\int_{-\infty}^{+\infty} \frac{d \beta}{\left(1+\beta^{2}\right)^{1 / 2}\left(1+\left|\xi^{\mathfrak{m}}-\beta\right|^{2}\right)^{1 / 2}} \leq C \frac{1+\log _{+}|\xi|}{\left(1+\xi^{2 \mathfrak{m}}\right)^{1 / 2}}
$$


where $\log _{+}|\xi|:=\max (0, \log |\xi|)$.

Using Lemma 3.2 we conclude therefore that

$$
\left|\mathbb{E} \hat{u}_{\varepsilon}^{(2 n)}(t, \xi)\right| \leq(2 n-1) ! ! \frac{C^{n} t^{n(2-\rho)} e^{t}}{(n !)^{2-\rho}} \frac{1+\log _{+}|\xi|}{\left(1+\xi^{2 \mathfrak{m}}\right)^{1 / 2}},
$$

and (4.6) follows by letting $a_{2 n}=(2 n-1) ! ! \frac{C^{n} t^{n(2-\rho)} e^{t}}{(n !)^{2-\rho}}$. We obtain

$$
\lim _{\varepsilon \rightarrow 0_{+}} \mathbb{E} \hat{u}_{\varepsilon}(t, \xi)=\sum_{n=0}^{+\infty} \bar{u}^{(2 n)}(t, \xi)
$$

where

$$
\begin{aligned}
\bar{u}^{(2 n)}(t, \xi):=(-\hat{R}(0))^{n} e^{t} \hat{u}_{0}(\xi) \sum_{\pi} \int_{\mathbb{R}} e^{i \beta t} d \beta \int \cdots \int \\
\quad \times\left\{\prod_{k=0}^{2 n}\left[1-i\left(\left|\xi-\sum_{j=1}^{k} \xi_{j}\right|^{\mathfrak{m}}-\beta\right)\right]\right\}^{-1} \prod_{(e f) \in \pi} \delta\left(\xi_{e}+\xi_{f}\right) d \xi_{e} d \xi_{f} .
\end{aligned}
$$

In what follows, we show that

$$
\bar{u}^{(2 n)}(t, \xi)=\mathbb{E} \hat{u}^{(2 n)}(t, \xi) .
$$

The expectation of multiple Stratonovich integrals is defined as follows:

$$
\mathbb{E}\left\{\prod_{j=1}^{2 n} d W\left(x_{j}\right)\right\}=\sum_{\pi} \prod_{(e f) \in \pi} \delta\left(x_{e}-x_{f}\right) d x_{e} d x_{f} .
$$

Upon integrating in all variables $x$, the first moment of $\hat{u}^{(2 n)}$ can therefore be written as

$$
\begin{aligned}
\mathbb{E} \hat{u}^{(2 n)}=(-i \sigma)^{2 n}(2 \pi)^{-2 n d} e^{t} \sum_{\pi} \int_{0}^{\infty} \cdots \int_{0}^{\infty} \int e^{i \beta t} d \beta \int \prod_{k=1}^{2 n} d \xi_{k} \\
\times\left\{\prod_{k=0}^{2 n}\left[1-i\left(\left|\xi-\sum_{j=1}^{k} \xi_{j}\right|^{\mathfrak{m}}-\beta\right)\right]\right\}^{-1} \hat{u}_{0}(\xi) \prod_{(e f) \in \pi} \delta\left(\xi_{e}+\xi_{f}\right),
\end{aligned}
$$

which gives exactly (3.33).

\section{4. $L^{2}$ convergence of the SPDE solution}

We now come to the series (1.9), which we claim is the solution to (1.6). The readers are referred to [1] for a more complete description of the relationship between the Stratonovich and Itô integrals and for additional details on the theory presented in this section.

We first prove that $\hat{u}(t, \cdot)$ in $(1.9)$ as a series is well-defined in the space $L^{2}\left(\Omega \times \mathbb{R}^{d}\right)$. Denote $\hat{u}^{(n)}=\mathcal{I}_{n}\left(f_{n}\right)$, where $\mathcal{I}_{n}$ denotes the $\mathrm{n}$-th order iterated Stratonovich integral, and $f_{n}$ is a n-parameter function, i.e.

$$
\mathcal{I}_{n}\left(f_{n}\right)=\int_{\mathbb{R}^{n d}} f_{n}\left(x_{1}, \ldots, x_{n}\right) d W\left(x_{1}\right) \ldots d W\left(x_{n}\right) .
$$


By the definition of the $L^{2}$ norm of multiple Stratonovich integral, we have

$$
\begin{aligned}
\mathbb{E}\left|\hat{u}^{(n)}\right|^{2} & =\mathbb{E}\left\{\mathcal{I}_{2 n}\left(f_{n} \otimes \bar{f}_{n}\right)\right\} \\
& =\mathbb{E} \int_{\mathbb{R}^{2 n d}} f_{n}\left(x_{1}, \ldots, x_{n}\right) \bar{f}_{n}\left(y_{1}, \ldots, y_{n}\right) d W\left(x_{1}\right) \ldots d W\left(x_{n}\right) d W\left(y_{1}\right) \ldots d W\left(y_{n}\right) .
\end{aligned}
$$

Taking the same approach as in the calculation of the first moment in (3.35), we obtain

$$
\begin{aligned}
& \mathbb{E}\left|\hat{u}^{(n)}(t, \xi)\right|^{2} \\
= & \left(\sigma^{n}(2 \pi)^{-d} e^{t}\right)^{2} \sum_{\pi_{2 n}} \iiint_{\mathbb{R}^{2}} e^{i\left(\beta_{1}-\beta_{2}\right) t} d \beta_{1} d \beta_{2} \hat{u}_{0}\left(\xi-\sum_{j=1}^{n} \xi_{j}^{(1)}\right) \hat{u}_{0}\left(\xi+\sum_{j=1}^{n} \xi_{j}^{(2)}\right) \\
& \times\left\{\prod_{k=0}^{n}\left[1-i\left(\left|\xi-\sum_{j=1}^{k} \xi_{j}^{(1)}\right|^{\mathfrak{m}}+\beta_{1}\right)\right] \prod_{k=0}^{n}\left[1+i\left(\left|\xi+\sum_{j=1}^{k} \xi_{j}^{(2)}\right|^{\mathfrak{m}}+\beta_{2}\right)\right]\right\}^{-1} \\
& \prod_{(e f) \in \pi} \delta\left(\xi_{e}+\xi_{f}\right) d \boldsymbol{\xi}^{(1)} d \boldsymbol{\xi}^{(2)} .
\end{aligned}
$$

We denote $d \boldsymbol{\xi}^{(l)}=d \xi_{1}^{(l)} \cdots d \xi_{n}^{(l)}, l=1,2 . \xi_{e}$ and $\xi_{f}$ are paired arguments in the graph comprised of $2 n$ arguments in total with their index $e$ and $f$.

The idea that we use to estimate the first moment applies here too. However, note that we have $\{k=n\} \in \mathcal{L}(\pi)$ for the crossing graphs defined in [2], and one of the terms $\left[1-i\left(\xi^{\mathfrak{m}}-\beta\right)\right]^{-1}$ in the function $F_{1}$ defined in (3.20) now becomes $[1-i(\mid \xi-$ $\left.\left.\left.\sum_{j=1}^{n} \xi_{j}^{(1)}\right|^{\mathfrak{m}}-\beta\right)\right]^{-1}$. Therefore, we have to make the following adjustments in the proof. Using the smoothness condition for the initial condition, we have

$$
\left|\frac{\hat{u}_{0}\left(\xi-\sum_{j=1}^{n} \xi_{j}^{(1)}\right)}{1-i\left(\left|\xi-\sum_{j=1}^{n} \xi_{j}^{(1)}\right|^{\mathfrak{m}}-\beta_{1}\right)}\right| \leq \frac{1}{\left(1+\left|\xi-\sum_{j=1}^{n} \xi_{j}^{(1)}\right|^{2 \mathfrak{m}}\right)^{1 / 2}} \frac{1}{\left(1+\beta_{1}^{2}\right)^{1 / 2}} .
$$

We use the term $\left[1+\left|\xi-\sum_{j=1}^{n} \xi_{j}^{(1)}\right|^{2 \mathfrak{m}}\right]^{-1 / 2}$ for the integration in $\xi_{n}$, and the term $\left[1+\beta_{1}^{2}\right]^{-1 / 2}$ together with $\left[1-i\left(\xi^{\mathfrak{m}}+\beta_{1}\right)\right]^{-1}$ indexed by $\{k=0\}$ in the first product in (4.3), and get

$$
\int_{-\infty}^{+\infty} \frac{d \beta_{1}}{\left(1+\beta_{1}^{2}\right)^{1 / 2}\left|1-i\left(\xi^{\mathfrak{m}}+\beta_{1}\right)\right|} \leq C \frac{1+\log _{+}|\xi|}{\left(1+\xi^{2 \mathfrak{m}}\right)^{1 / 2}}
$$

We also use the smoothness condition for the initial condition and obtain another $\left(1+\log _{+}|\xi|\right) /\left(1+\xi^{2 \mathfrak{m}}\right)^{1 / 2}$ from the integration in $\beta_{2}$. Finally, we obtain the estimate

$$
\mathbb{E}\left|\hat{u}^{(n)}(t, \xi)\right|^{2} \leq(2 n-1) ! ! \frac{C^{n} t^{n(2-\rho)} e^{t}}{(n !)^{2-\rho}} \frac{1+\log _{+}|\xi|}{\left(1+\xi^{2 \mathfrak{m}}\right)^{1 / 2}}
$$

for any $\rho \in\left(\frac{d}{\mathfrak{m}}, 1\right)$. This implies that the iterated Stratonovich integral $\hat{u}^{(n)}$ is indeed well-defined. Integrating in $\xi$ and summing the above bound over $n$ gives

$$
\left(\mathbb{E} \int|\hat{u}(t, \xi)|^{2} d \xi\right)^{1 / 2} \leq \sum_{n \geq 0}\left(\mathbb{E} \int\left|\hat{u}^{(n)}(t, \xi)\right|^{2} d \xi\right)^{1 / 2}<\infty
$$


and the $L^{2}\left(\Omega \times \mathbb{R}^{d}\right)$ convergence of (1.9) follows. In fact, by first multiplying $\xi^{\mathfrak{m}}$ with (4.6) and performing the integration and summation we can further obtain

$$
\mathbb{E} \int \xi^{\mathfrak{m}}|\hat{u}(t, \xi)|^{2} d \xi<\infty .
$$

\section{Uniqueness of the SPDE solution}

Let us now provide the rigorous definition for the operator $\mathcal{H}$. Suppose $f(t, \xi)$ is a sum of iterated Stratonovich integrals

$$
f(t, \xi)=\sum_{n \geq 0} \mathcal{I}_{n}\left(f_{n}(t, \xi, \cdot)\right) .
$$

We define

$$
\mathcal{H} f(t, \xi)=\sum_{n \geq 1} \mathcal{I}_{n}\left((\mathcal{H} f)_{n}(t, \xi, \cdot)\right)
$$

where

$$
(\mathcal{H} f)_{n+1}\left(t, \xi, x, x_{1}, \cdots, x_{n}\right):=(-i \sigma)(2 \pi)^{-d} \int_{0}^{t} \int e^{i(t-s) \xi^{\mathrm{m}}} e^{i\left(\xi_{0}-\xi\right) x} f_{n}\left(s, \xi_{0}, x_{1}, \cdots, x_{n}\right) d \xi_{0} d s .
$$

One can check that under this definition $\mathcal{H} \hat{u}=\sum_{n \geq 1} \hat{u}^{(n)}=\hat{u}-\hat{u}^{(0)}$. The Duhamel solution (1.9) is therefore a solution to the equation (1.6).

We can also define

$$
\mathcal{J} f(t, \xi)=\sum_{n \geq 0} \mathcal{I}_{n+1}\left((\mathcal{J} f)_{n+1}(t, \xi, \cdot)\right)
$$

where

$$
(\mathcal{J} f)_{n+1}\left(t, \xi, x, x_{1}, \cdots, x_{n}\right):=e^{-i \xi x} \int e^{i \xi_{0} x} f_{n}\left(s, \xi_{0}, x_{1}, \cdots, x_{n}\right) d \xi_{0} .
$$

For the Duhamel solution (1.9), we have

$$
\begin{aligned}
& \mathbb{E}\left|\mathcal{I}_{n+1}\left((\mathcal{J} \hat{u})_{n+1}(t, \xi)\right)\right|^{2} \\
& =\left(\sigma^{n}(2 \pi)^{-d} e^{t}\right)^{2} \sum_{\pi_{2 n}} \iiint_{\mathbb{R}^{2}} e^{i\left(\beta_{1}-\beta_{2}\right) t} d \beta_{1} d \beta_{2} \hat{u}_{0}\left(\xi-\sum_{j=1}^{n+1} \xi_{j}^{(1)}\right) \overline{\hat{u}}_{0}\left(\xi+\sum_{j=1}^{n+1} \xi_{j}^{(2)}\right) \\
& \quad \times\left\{\prod_{k=1}^{n+1}\left[1-i\left(\left|\xi-\sum_{j=1}^{k} \xi_{j}^{(1)}\right|^{\mathfrak{m}}+\beta_{1}\right)\right] \prod_{k=1}^{n+1}\left[1+i\left(\left|\xi+\sum_{j=1}^{k} \xi_{j}^{(2)}\right|^{\mathfrak{m}}+\beta_{2}\right)\right]\right\}^{-1} \\
& \quad \times \prod_{(e f) \in \pi} \delta\left(\xi_{e}+\xi_{f}\right) d \boldsymbol{\xi}^{(1)} d \boldsymbol{\xi}^{(2)} .
\end{aligned}
$$

Although it looks a little different from (4.3), it can be estimated in the same way as

$$
\left(\mathbb{E}\left|\hat{u}^{(n)}\right|^{2}\right)^{1 / 2}<(2 n-1) ! ! \frac{C^{n} t^{n(2-\rho)} e^{t}}{(n !)^{2-\rho}} \frac{1+\log _{+}|\xi|}{\left(1+\xi^{2 \mathfrak{m}}\right)^{1 / 2}},
$$

which upon summation in $n$ implies that $\mathbb{E}|\hat{u}(t, \xi)|^{2}$ is uniformly bounded for all $\xi \in \mathbb{R}^{d}$. 
Note that $\mathcal{H} \hat{u}(t, \xi)=(-i \sigma)(2 \pi)^{-d} \int_{0}^{t} e^{i(t-s) \xi^{\mathrm{m}}}(\mathcal{J} \hat{u})(s, \xi) d s . \quad \hat{u}(t, \xi)$ is therefore a solution to the equation

$$
\left(i \frac{\partial}{\partial t}+\xi^{\mathfrak{m}}\right) \hat{u}(t, \xi)=\sigma(2 \pi)^{-d} \mathcal{J} \hat{u}(t, \xi) .
$$

Now we prove that this equation preserves mass. Multiplying this equation by $\overline{\hat{u}}(t, \xi)$, and integrating in $\xi$ and over the probability space $\Omega$ gives

$$
\frac{i}{2} \frac{\partial}{\partial t} \mathbb{E} \int|\hat{u}|^{2} d \xi+\mathbb{E} \int \xi^{\mathfrak{m}}|\hat{u}|^{2} d \xi=\sigma(2 \pi)^{-d} \mathbb{E} \int(\mathcal{J} \hat{u}) \overline{\hat{u}} d \xi
$$

The right hand side of this equation can be written out explicitly as

$$
\begin{aligned}
\mathbb{E} \int(\mathcal{J} \hat{u}) \overline{\hat{u}} d \xi & =\int \sum_{n, m} \mathbb{E}\left\{\mathcal{I}_{n+1}\left(e^{-i \xi x} \int e^{i \xi_{1} x} f_{n}\left(t, \xi_{1}\right) d \xi_{1}\right) \mathcal{I}_{m}\left(\bar{f}_{m}(t, \xi)\right)\right\} d \xi \\
& =\int \sum_{n, m} \int\left(e^{-i \xi x} \int e^{i \xi_{1} x} f_{n}\left(t, \xi_{1}\right) d \xi_{1} \bar{f}_{m}(t, \xi) \sum_{\pi} \prod_{(e f) \in \pi} \delta\left(x_{e}-x_{f}\right) d \mathbf{x}\right) d \xi \\
& =\sum_{n, m} \sum_{\pi} \int e^{i \xi_{1} x} f_{n}\left(t, \xi_{1}\right) d \xi_{1} \int e^{-i \xi x} \bar{f}_{m}(t, \xi) d \xi \prod_{(e f) \in \pi} \delta\left(x_{e}-x_{f}\right) d \mathbf{x} \\
& =\sum_{n, m} \sum_{\pi} \int \check{f}_{n}(t, x) \bar{f}_{m}(t, x) \prod_{(e f) \in \pi} \delta\left(x_{e}-x_{f}\right) d \mathbf{x},
\end{aligned}
$$

which is real-valued because of the symmetry of this summation. Extracting the imaginary part from both sides of (5.9) gives

$$
\frac{\partial}{\partial t} \mathbb{E} \int|\hat{u}(t, \xi)|^{2} d \xi=0
$$

Finally, we define the space $M$ in which the equation (5.8) admits a unique solution. In light of the equation (5.9), $M$ consists of sum of iterated Stratonovich integrals $f(t, \xi)=\sum_{n \geq 0} \mathcal{I}_{n}\left(f_{n}(t, \xi, \cdot)\right)$ such that

1. $f(t, \xi) \in L^{2}\left(\Omega \times \mathbb{R}^{d}\right)$,

2. $\mathcal{J} f \in L^{2}(\Omega)$,

3. $|\xi|^{\frac{\mathfrak{m}}{2}} f(t, \xi) \in L^{2}\left(\Omega \times \mathbb{R}^{d}\right)$.

As a reminder, defining the sum of iterated Stratonovich integrals $f$ as in (5.1), we have that

$$
f=\sum_{n \geq 0} \mathcal{I}_{n}\left(f_{n}\right)=\sum_{m \geq 0} I_{m}\left(g_{m}\right)
$$

where

$$
g_{m}(t, \xi, x)=\sum_{k \geq 0} \frac{(m+2 k) !}{m ! k ! 2^{k}} \int_{\mathbb{R}^{k d}} f_{m+2 k}\left(t, \xi, \mathbf{x}_{m}, \mathbf{y}_{k}^{\otimes 2}\right) d \mathbf{y}
$$

Here, $\mathbf{y} \otimes \mathbf{y} \equiv(y, y)$, and

$$
I_{m}\left(g_{m}\right):=\int_{\mathbb{R}^{m d}} g_{m}\left(t, \xi, x_{1}, \cdots, x_{m}\right) d W\left(x_{1}\right) \cdots d W\left(x_{m}\right)
$$


denotes the iterated Itô integral. The $L^{2}$ norm of $f$ can then be computed using the orthogonality of the Wiener Chaos expansion as

$$
\|f\|_{L^{2}(\Omega)}=\left(\sum_{m \geq 0} m ! \int\left(\sum_{k \geq 0} \frac{(m+2 k) !}{m ! k ! 2^{k}} \int_{\mathbb{R}^{k d}}\left|f_{m+2 k}\right|\left(t, \xi, \mathbf{x}_{m}, \mathbf{y}_{k}^{\otimes 2}\right) d \mathbf{y}\right)^{2} d \mathbf{x}\right)^{\frac{1}{2}}<\infty .
$$

The readers are referred to [1] for more details.

It is easy to verify that the space defined above is dense in $L^{2}\left(\Omega \times \mathbb{R}^{d}\right)$. Denote the space consisting of all functions that satisfy condition (1) and (2) by $\tilde{M}$. In fact, any function $f(\xi) \in L^{2}\left(\Omega \times \mathbb{R}^{d}\right)$ can be written as its Wiener Chaos expansion

$$
f(\xi)=\sum_{m \geq 0} I_{m}\left(g_{m}\left(\xi, \mathbf{x}_{m}\right)\right) .
$$

Each $g_{m}$ can be approximated by a function $f_{m}^{k}$, which vanishes in a set of measure at most $k^{-1}$ in the vicinity of the measure 0 set of diagonals given by the support of the distributions $\delta\left(x_{e}-x_{f}\right)$. By the change of change of coordinates in (5.13), we have $g_{m}^{(k)}=f_{m}^{(k)}$, so that the Itô and Stratonovich integrals agree. Define

$$
f^{(k)}(\xi)=\sum_{m \geq 0} \mathcal{I}_{m}\left(f_{m}^{(k)}\right) .
$$

Using formula (5.15), we may verify that

$$
\left\|\mathcal{J} f^{(k)}\right\|_{L^{2}(\Omega)}<\infty
$$

and

$$
\lim _{k \rightarrow \infty}\left\|f^{(k)}(\xi)-f(\xi)\right\|_{L^{2}\left(\Omega \times \mathbb{R}^{d}\right)}=0 .
$$

We have shown that $\tilde{M}$ is dense in $L^{2}\left(\Omega \times \mathbb{R}^{d}\right)$. Since $M$ is dense in $\tilde{M}$, it is also dense in $L^{2}\left(\Omega \times \mathbb{R}^{d}\right)$.

\section{General moment convergence}

We now extend the result of Section 3 to general moment convergence. It suffices to prove that for all $\left(t_{1}, \xi^{(1)}\right), \cdots,\left(t_{r}, \xi^{(r)}\right)$, we have

$$
\lim _{\varepsilon \rightarrow 0} \mathbb{E}\left\{\hat{u}_{\varepsilon}\left(t_{1}, \xi^{(1)}\right) \cdots \hat{u}_{\varepsilon}\left(t_{r}, \xi^{(r)}\right)\right\}=\mathbb{E}\left\{\hat{u}\left(t_{1}, \xi^{(1)}\right) \cdots \hat{u}\left(t_{r}, \xi^{(r)}\right)\right\} .
$$

We will take the same approach as we did for proving the first moment convergence. Specifically, we shall rewrite $\hat{u}_{\varepsilon}$ as the expansion (2.1), and show that every cross moment

$$
\mathcal{I}_{\varepsilon}(\boldsymbol{n}):=\mathbb{E}\left\{\hat{u}_{\varepsilon}^{\left(n_{1}\right)}\left(t_{1}, \xi^{(1)}\right) \cdots \hat{u}_{\varepsilon}^{\left(n_{r}\right)}\left(t_{r}, \xi^{(r)}\right)\right\}
$$

converges, where $\boldsymbol{n}=\left(n_{1}, \cdots, n_{r}\right)$. We point out that since all $\mathcal{I}_{\varepsilon}(\boldsymbol{n})$ are the expectation of a product of Gaussian variables, the terms for which $|\boldsymbol{n}|:=\sum_{l=1}^{r} n_{l}$ are odd are equal to 0 . Using equation $(2.5)$ and (3.3) we may write $\mathcal{I}_{\varepsilon}(\boldsymbol{n})$ explicitly as

$$
\mathcal{I}_{\varepsilon}(\boldsymbol{n})=(-i)^{|\boldsymbol{n}|} \exp \left(\sum_{l=1}^{r} t_{l}\right) \sum_{\pi} \int_{\mathbb{R}^{|\boldsymbol{n}| d}} \exp \left\{i \sum_{l=1}^{r} \beta_{l} t_{l}\right\} \prod_{l=1}^{r} d \beta_{l} \int \ldots \int \prod_{l=1}^{r} d \boldsymbol{\xi}^{(l)}
$$




$$
\begin{aligned}
& \times \prod_{l=1}^{r} \hat{u}_{0}\left(\xi^{(l)}-\sum_{k=1}^{n_{l}} \xi_{k}^{(l)}\right) \prod_{(e f) \in \pi} \hat{R}\left(\varepsilon \xi_{e}\right) \delta\left(\xi_{e}+\xi_{f}\right) \\
& \times\left\{\prod_{l=1}^{r} \prod_{k=0}^{n_{l}}\left[1-i\left(\left|\xi^{(l)}-\sum_{j=1}^{k} \xi_{j}^{(l)}\right|^{\mathfrak{m}}-\beta\right)\right]\right\}^{-1} .
\end{aligned}
$$

Here, we define $d \boldsymbol{\xi}^{(l)}:=d \xi_{1}^{(l)} \cdots d \xi_{n_{l}}^{(l)}$, and denote the pair of vertices by $(e f)$. As $\varepsilon \rightarrow 0$, $\mathcal{I}_{\varepsilon}(\boldsymbol{n})$ converges to

$$
\begin{aligned}
\mathcal{I}(\boldsymbol{n}):=\left(-i \sigma(2 \pi)^{-d}\right)^{|\boldsymbol{n}|} \sum_{\pi} \int_{\mathbb{R}^{\mid}|\boldsymbol{n} d|} \exp \left\{i \sum_{l=1}^{r} \beta_{l} t_{l}\right\} \prod_{l=1}^{r} d \beta_{l} \int \cdots \int \prod_{l=1}^{r} d \boldsymbol{\xi}^{(l)} \\
\times \prod_{l=1}^{r} \hat{u}_{0}\left(\xi^{(l)}-\sum_{k=1}^{n_{l}} \xi_{k}^{(j)}\right) \prod_{(e f) \in \pi} \delta\left(\xi_{e}+\xi_{f}\right) \\
\quad \times\left\{\prod_{l=1}^{r} \prod_{k=0}^{n_{l}}\left[1-i\left(\left|\xi^{(l)}-\sum_{j=1}^{k} \xi_{j}^{(l)}\right|^{\mathfrak{m}}-\beta_{l}\right)\right]\right\}^{-1} \\
=\mathbb{E}\left\{\hat{u}^{\left(n_{1}\right)}\left(t_{1}, \xi^{(1)}\right) \cdots \hat{u}^{\left(n_{r}\right)}\left(t_{r}, \xi^{(r)}\right)\right\},
\end{aligned}
$$

which are the cross moments that appear on the right hand side of (6.1).

Now we show that moving the passage to the limit of $\varepsilon \rightarrow 0_{+}$inside the summation is legitimate. Reproducing the work for estimating the first order moment in Section 3 yields

$$
\left|\mathcal{I}_{\varepsilon}(\boldsymbol{n})\right| \leq(|\boldsymbol{n}|-1) ! ! \frac{\left.\left(C t^{2-\rho}\right)^{\frac{|\boldsymbol{n}|}{2}} e^{r T}\right)}{\left(\frac{|\boldsymbol{n}|}{2} !\right)^{2-\rho}},
$$

assuming $0 \leq t_{1}, \ldots, t_{r} \leq T$, where the constant $C$ is independent of $\varepsilon \in(0,1],|\boldsymbol{n}|$, and $r$. The summation of $\left(\begin{array}{c}N+r-1 \\ r-1\end{array}\right)$ non-negative integer-valued multi-indices satisfying equation $|\boldsymbol{n}|=N$ is estimated as

$$
\left|\sum_{|\boldsymbol{n}|=N} \mathcal{I}_{\varepsilon}(\boldsymbol{n})\right| \leq c_{N}
$$

where

$$
c_{N}:=\left(\begin{array}{c}
N+r-1 \\
r-1
\end{array}\right)(N-1) ! ! \frac{\left(C T^{2-\rho}\right)^{\frac{N}{2}} e^{r T}}{\left(\frac{N}{2} !\right)^{2-\rho}} .
$$

The convergence of $\sum_{N=1}^{+\infty} c_{N}$ is easy to verify.

As a result, we conclude that

$$
\lim _{\varepsilon \rightarrow 0_{+}} \mathbb{E}\left\{\hat{u}_{\varepsilon}\left(t_{1}, \xi^{(1)}\right) \cdots \hat{u}_{\varepsilon}\left(t_{r}, \xi^{(r)}\right)\right\}=\sum \lim _{\varepsilon \rightarrow 0_{+}} \mathcal{I}_{\varepsilon}(\boldsymbol{n})=\sum \mathcal{I}(\boldsymbol{n})=\mathbb{E}\left\{\hat{u}\left(t_{1}, \xi^{(1)}\right) \cdots \hat{u}\left(t_{r}, \xi^{(r)}\right)\right\}
$$




\section{Weak convergence}

The sufficient condition to prove the weak convergence of $\hat{u}_{\varepsilon}(t, \xi)$ in $C\left([0,+\infty), \mathcal{S}^{\prime}\left(\mathbb{R}^{d}\right)\right)$ is tightness and the convergence of finite dimensional distribution.

In order to prove the tightness of $\left\{\hat{u}_{\varepsilon}(t, \xi)\right\}$ over $C\left([0,+\infty), \mathcal{S}^{\prime}\left(\mathbb{R}^{d}\right)\right)$, it suffices (by [4]) to prove the tightness of $\left\{u_{\varepsilon}^{\phi}(t):=\left\langle\hat{u}_{\varepsilon}(t, \cdot), \phi\right\rangle_{L^{2}\left(\mathbb{R}^{d}\right)}, t \geq 0\right\}$ for an arbitrary $\phi \in \mathcal{S}\left(\mathbb{R}^{d}\right)$, which, by Kolmogorov's theorem, follows from the following result.

Proposition 7.1. For $T>0$ and $\phi \in \mathcal{S}\left(\mathbb{R}^{d}\right)$ there exists a constant $C>0$ such that

$$
\mathbb{E}\left|u_{\varepsilon}^{\phi}(t)-u_{\varepsilon}^{\phi}(s)\right|^{2} \leq C(t-s)^{2},
$$

uniformly in $s, t \in[0, T]$ and $\varepsilon>0$.

Proof. From equation (1.2), we obtain

$$
u_{\varepsilon}^{\phi}(t)-u_{\varepsilon}^{\phi}(s)=i \int_{s}^{t} u_{\varepsilon}^{\phi_{1}}(\tau) d \tau+(-i) \varepsilon^{-\frac{d}{2}} \int_{s}^{t} \iint \hat{q}(\zeta) \hat{u}_{\varepsilon}^{\phi}\left(\tau, \xi-\varepsilon^{-1} \zeta\right) d \zeta d \xi d \tau,
$$

where $\phi_{1}(\xi):=\xi^{\mathfrak{m}} \phi(\xi)$. Define

$$
\begin{aligned}
v_{\varepsilon}^{\phi}(\tau):= & \sum_{n \geq 0} v_{\varepsilon}^{n, \phi}(\tau) \\
v_{\varepsilon}^{n, \phi}(\tau):=( & -i)^{n+1} \varepsilon^{-\frac{d(n+1)}{2}} e^{\tau} \int_{\mathbb{R}} e^{i \beta \tau} d \beta \int \ldots \int \prod_{k=0}^{n} \hat{q}\left(\xi_{k}\right) d \xi_{k} \int_{\mathbb{R}^{d}} d \xi \\
& \times\left\{\prod_{k=0}^{n}\left[1-i\left(\left|\xi-\varepsilon^{-1} \sum_{j=0}^{k} \xi_{j}\right|^{\mathfrak{m}}-\beta\right)\right]\right\}^{-1} \hat{u}_{0}\left(\xi-\varepsilon^{-1} \sum_{j=0}^{n} \xi_{j}\right) \phi(\xi) .
\end{aligned}
$$

We then have

$$
u_{\varepsilon}^{\phi}(t)-u_{\varepsilon}^{\phi}(s)=i \int_{s}^{t} u_{\varepsilon}^{\phi_{1}}(\tau) d \tau+\int_{s}^{t} v_{\varepsilon}^{\phi}(\tau) d \tau .
$$

Mimicking the proof in Section 4, we argue that for all $\tau \in[0, T]$, we have $\mathbb{E}\left|u_{\varepsilon}^{\phi_{1}}(\tau)\right|^{2} \leq C$ and $\mathbb{E}\left|v_{\varepsilon}^{\phi}(\tau)\right|^{2} \leq C$ for a constant $C$ independent of $\varepsilon \in(0,1]$. Applying the Cauchy-Schwarz inequality to the right hand side of (7.4) leads to (7.1).

Using (2.10) for any $\phi \in \mathcal{S}\left(\mathbb{R}^{d}\right)$, we can write

$$
\begin{aligned}
& \left\langle\hat{u}^{(n)}(t, \cdot), \phi\right\rangle-\left\langle\hat{u}^{(n)}(s, \cdot), \phi\right\rangle \\
= & \int_{s}^{t} e^{\tau} d \tau \int_{\mathbb{R}} e^{-i \beta \tau} d \beta \int \ldots \int \prod_{k=1}^{n} d \xi_{k} \int d \xi \\
& \times\left\{\prod_{k=0}^{n}\left[1-i\left(\left|\xi-\sum_{j=1}^{k} \xi_{j}\right|^{\mathfrak{m}}+\beta\right)\right]\right\}^{-1} \hat{u}_{0}\left(\xi-\sum_{j=1}^{n} \xi_{j}\right) \phi(\xi) \prod_{j=1}^{n} e^{-i \xi_{j} x_{j}} \circ \prod_{j=1}^{n} d W\left(x_{j}\right) \\
& -i \int_{s}^{t} e^{\tau} d \tau \int_{\mathbb{R}} \beta e^{-i \beta \tau} d \beta \int \ldots \int \prod_{k=1}^{n} d \xi_{k} \int d \xi \\
& \times\left\{\prod_{k=0}^{n}\left[1-i\left(\left|\xi-\sum_{j=1}^{k} \xi_{j}\right|^{\mathfrak{m}}+\beta\right)\right]\right\}^{-1} \hat{u}_{0}\left(\xi-\sum_{j=1}^{n} \xi_{j}\right) \phi(\xi) \prod_{j=1}^{n} e^{-i \xi_{j} x_{j}} \circ \prod_{j=1}^{n} d W\left(x_{j}\right) .
\end{aligned}
$$


Applying the technique that we used in Section 4, we check that for any $T>0$, we have

$$
\mathbb{E}\left|\left\langle\hat{u}^{(n)}(t, \cdot), \phi\right\rangle-\left\langle\hat{u}^{(n)}(s, \cdot), \phi\right\rangle\right|^{2} \leq(C T)^{2}(t-s)^{2}(n !)^{(1-\rho)}, \forall n \geq 1, s, t \in[0, T]
$$

for some constant $C>0$ independent of $n$. This in turn implies that

$$
\mathbb{E}|\langle\hat{u}(t, \cdot), \phi\rangle-\langle\hat{u}(s, \cdot), \phi\rangle|^{2} \leq C(t-s)^{2}, \forall n \geq 1
$$

on any compact set. By the Kolmogorov-Chentsov Theorem, we have that $\langle\hat{u}(t, \cdot), \phi\rangle$ is continuous almost surely.

It remains to prove the convergence of the finite dimensional distribution of $\hat{u}_{\varepsilon}$, i.e., the convergence of distributions of $\left(\hat{u}_{\varepsilon}\left(t_{1}, \xi^{(1)}\right), \cdots, \hat{u}_{\varepsilon}\left(t_{r}, \xi^{(r)}\right)\right)$ for an arbitrary $r \geq 1, t_{1}, \cdots, t_{r} \geq 0, \xi^{(1)}, \cdots, \xi^{(r)} \in \mathbb{R}^{d}$. For simplicity we consider only the case $r=1$, as it is easy to generalize to the case for arbitrary $r$. Since we have already had moment convergence, it suffices to verify the determinacy of the distributions by their moments. In fact, we point out that the estimate (6.5) still holds if we replace some of the terms in (6.2) with their complex conjugates. Therefore, for $n$ being even, we have

$$
\mathbb{E}|\hat{u}(t, \xi)|^{n} \leq \sum_{N=0}^{+\infty}\left(\begin{array}{c}
N+n-1 \\
n-1
\end{array}\right)(N-1) ! ! \frac{\left(C t^{2-\rho}\right)^{\frac{N}{2}} e^{n t}}{\left(\frac{N}{2} !\right)^{2-\rho}} .
$$

Using Stirling's formula we can easily obtain that

$$
\left(\begin{array}{c}
N+n-1 \\
n-1
\end{array}\right) \leq C\left(1+\frac{n-1}{N}\right)^{N}\left(1+\frac{N}{n-1}\right)^{n-1} .
$$

Plugging this into equation (7.8) therefore gives

$$
\begin{aligned}
\mathbb{E}|\hat{u}(t, \xi)|^{n} \leq C 2^{n-1} e^{n t} \sum_{N=0}^{n-1}\left(1+\frac{n-1}{N}\right)^{N}(N-1) ! ! \frac{\left(C t^{2-\rho}\right)^{\frac{N}{2}}}{\left(\frac{N}{2} !\right)^{2-\rho}} \\
+C e^{n t} \sum_{N=n}^{+\infty}\left(1+\frac{N}{n-1}\right)^{n-1}(N-1) ! ! \frac{\left(C t^{2-\rho}\right)^{\frac{N}{2}}}{\left(\frac{N}{2} !\right)^{2-\rho}}
\end{aligned}
$$

The first term is bounded by $C n^{n}$ while the second by a constant $C$ independent of $n$. Therefore we have

$$
\sum_{n=1}^{+\infty} \frac{1}{\left[\mathbb{E}|\hat{u}(t, \xi)|^{2 n}\right]^{1 / 2 n}} \geq C \sum_{n=1}^{+\infty} \frac{1}{n^{1 / 2}}=+\infty,
$$

and the uniqueness follows from Carleman's condition.

Weak convergence of $\left\{\hat{u}_{\varepsilon}(t, \xi)\right\}$ follows from the convergence of finite dimensional distribution and tightness. Back in the spatial space, by the Plancherel theorem, we have $\langle u(t, \cdot), \phi\rangle=\langle\hat{u}(t, \cdot), \hat{\phi}\rangle$. Hence, the process $\left\{u_{\varepsilon}(t, x)\right\}$ converges in law over $C\left([0,+\infty) ; \mathcal{S}^{\prime}\left(\mathbb{R}^{d}\right)\right)$ to $\{u(t, x)\}$.

Acknowledgment. The authors would like to thank Tomasz Komorowski for useful discussions during the preparation of the manuscript. This paper was partially funded by AFOSR Grant NSSEFF- FA9550-10-1-0194 and NSF grant DMS-1108608. 


\section{REFERENCES}

[1] G. Bal, Convergence to SPDEs in Stratonovich form, Comm. Math. Phys., 212(2), 457-477, 2009.

[2] G. Bal, Homogenization with large spatial random potential, Multiscale Model. Simul., 8(4), 1484-1510, 2010.

[3] L. Erdös and H.T. Yau, Linear Boltzmann equation as the weak coupling limit of a random Schrödinger equation, Comm. Pure Appl. Math., 53(6), 667-735, 2000.

[4] I. Mitoma, Tightness of probabilities on $C\left([0,1] ; \mathcal{S}^{\prime}\right)$ and $D\left([0,1] ; \mathcal{S}^{\prime}\right)$, Ann. Prob., 11(4), 989999, 1983.

[5] T. Komorowski and E. Nieznaj, On the asymptotic behavior of solutions of the heat equation with a random, long-range correlated potential, Pot. Anal., 33(2), 175-197, 2010.

[6] E. Pardoux and A. Piatnitski, Homogenization of a singular random one dimensional PDE, GAKUTO Internat. Ser. Math. Sci. Appl., 24, 291-303, 2006.

[7] N. Zhang and G. Bal, Homogenization of the Schroedinger equation with large, random potential, Stoch. Dyn., 14(1), 1350013, 2014. 